\title{
SOME INEQUALITIES OF HERMITE-HADAMARD TYPE FOR TRIGONOMETRICALLY $\rho$-CONVEX FUNCTIONS
}

\author{
SILVESTRU SEVER DRAGOMIR ${ }^{1,2}$
}

\begin{abstract}
In this paper we establish several Hermite-Hadamard type integral inequalities for trigonometrically $\rho$-convex functions. Applications for special means are provided as well.

Keywords: Mathematical Analysis
\end{abstract}

\section{IntRoduction}

The following integral inequality

$$
f\left(\frac{a+b}{2}\right) \leq \frac{1}{b-a} \int_{a}^{b} f(t) d t \leq \frac{f(a)+f(b)}{2},
$$

which holds for any convex function $f:[a, b] \rightarrow \mathbb{R}$, is well known in the literature as the Hermite-Hadamard inequality.

There is an extensive amount of literature devoted to this simple and nice result which has many applications in the Theory of Special Means and in Information Theory for divergence measures, for which we would like to refer the reader to the monograph [8], the recent survey paper [7] and the references therein.

Let $I$ be a finite or infinite open interval of real numbers and $\rho>0$.

In the following we present the basic definitions and results concerning the class of trigonometrically $\rho$-convex function, see for example [10], [11] and [3], [5], [6], [9], [12], [13] and [14].

Following [1], we say that a function $f: I \rightarrow \mathbb{R}$ is trigonometrically $\rho$-convex on $I$ if for any closed subinterval $[a, b]$ of $I$ with $0<b-a<\frac{\pi}{\rho}$ we have

$$
f(x) \leq \frac{\sin [\rho(b-x)]}{\sin [\rho(b-a)]} f(a)+\frac{\sin [\rho(x-a)]}{\sin [\rho(b-a)]} f(b)
$$

for all $x \in[a, b]$.

If the inequality (1.2) holds with " $\geq$ ", then the function will be called trigonometrically $\rho$-concave on $I$.

Geometrically speaking, this means that the graph of $f$ on $[a, b]$ lies nowhere above the $\rho$-trigonometric function determined by the equation

$$
H(x)=H(x ; a, b, f):=A \cos (\rho x)+B \sin (\rho x)
$$

where $A$ and $B$ are chosen such that $H(a)=f(a)$ and $H(b)=f(b)$.

1991 Mathematics Subject Classification. 26D15, 26D10, 26D07, 26A33.

Key words and phrases. Convex functions, Trigonometrically $\rho$-Convex Functions, HermiteHadamard inequalities, Integral inequalities. 
If we take $x=(1-t) a+t b \in[a, b], t \in[0,1]$, then the condition (1.2) becomes

$$
f((1-t) a+t b) \leq \frac{\sin [\rho(1-t)(b-a)]}{\sin [\rho(b-a)]} f(a)+\frac{\sin [\rho t(b-a)]}{\sin [\rho(b-a)]} f(b)
$$

for any $t \in[0,1]$.

We have the following properties of trigonometrically $\rho$-convex on $I$, [1]

(i) A trigonometrically $\rho$-convex function $f: I \rightarrow \mathbb{R}$ has finite right and left derivatives $f_{+}^{\prime}(x)$ and $f_{-}^{\prime}(x)$ at every point $x \in I$ and $f_{-}^{\prime}(x) \leq f_{+}^{\prime}(x)$. The function $f$ is differentiable on $I$ with the exception of an at most countable set.

(ii) A necessary and sufficient condition for the function $f: I \rightarrow \mathbb{R}$ to be trigonometrically $\rho$-convex function on $I$ is that it satisfies the gradient inequality

$$
f(y) \geq f(x) \cos [\rho(y-x)]+K_{x, f} \sin [\rho(y-x)]
$$

for any $x, y \in I$ where $K_{x, f} \in\left[f_{-}^{\prime}(x), f_{+}^{\prime}(x)\right]$. If $f$ is differentiable at the point $x$ then $K_{x, f}=f^{\prime}(x)$.

(iii) A necessary and sufficient condition for the function $f$ to be a trigonometrically $\rho$-convex in $I$, is that the function

$$
\varphi(x)=f^{\prime}(x)+\rho^{2} \int_{a}^{x} f(t) d t
$$

is nondecreasing on $I$, where $a \in I$.

(iv) Let $f: I \rightarrow \mathbb{R}$ be a two times continuously differentiable function on $I$. Then $f$ is trigonometrically $\rho$-convex on $I$ if and only if for all $x \in I$ we have

$$
f^{\prime \prime}(x)+\rho^{2} f(x) \geq 0
$$

For other properties of trigonometrically $\rho$-convex functions, see [1].

As general examples of trigonometrically $\rho$-convex functions we can give the indicator function

$$
h_{F}(\theta):=\limsup _{r \rightarrow \infty} \frac{\log \left|F\left(r e^{i \theta}\right)\right|}{r^{\rho}}, \theta \in(\alpha, \beta),
$$

where $F$ is an entire function of order $\rho \in(0, \infty)$.

If $0<\beta-\alpha<\frac{\pi}{\rho}$, then, it was shown in 1908 by Phragmén and Lindelöf, see [10], that $h_{F}$ is trigonometrically $\rho$-convex on $(\alpha, \beta)$.

Using the condition (1.5) one can also observe that any nonnegative twice differentiable and convex function on $I$ is also trigonometrically $\rho$-convex on $I$ for any $\rho>0$.

There exists also concave functions on an interval that are trigonometrically $\rho$-convex on that interval for some $\rho>0$.

Consider for example $f(x)=\cos x$ on the interval $\left[-\frac{\pi}{2}, \frac{\pi}{2}\right]$, then

$$
f^{\prime \prime}(x)+\rho^{2} f(x)=-\cos x+\rho^{2} \cos x=\left(\rho^{2}-1\right) \cos x
$$

which shows that it is trigonometrically $\rho$-convex on the interval $\left[-\frac{\pi}{2}, \frac{\pi}{2}\right]$ for all $\rho>1$ and trigonometrically $\rho$-concave for $\rho \in(0,1)$.

In this paper we establish several Hermite-Hadamard type integral inequalities for trigonometrically $\rho$-convex functions. Applications for special means are provided as well. 


\section{Some Hermite-Hadamard Type Inequalities}

We start with the following lemma of interest in itself:

Lemma 1. Assume that the function $f: I \rightarrow \mathbb{R}$ is trigonometrically $\rho$-convex on I. Then for any $a, b \in I$ with $0<b-a<\frac{\pi}{\rho}$ and $x \in[a, b]$ we have

$$
\begin{aligned}
f\left(\frac{a+b}{2}\right) \cos \left[\rho\left(x-\frac{a+b}{2}\right)\right] \leq \frac{1}{2}[f(x)+f(a+b-x)] \\
\leq \frac{f(a)+f(b)}{2} \frac{\cos \left[\rho\left(x-\frac{a+b}{2}\right)\right]}{\cos \left[\frac{\rho(b-a)}{2}\right]}
\end{aligned}
$$

Proof. From (1.2) we have by replacing $x$ with $a+b-x$ that

$$
f(a+b-x) \leq \frac{\sin [\rho(x-a)]}{\sin [\rho(b-a)]} f(a)+\frac{\sin [\rho(b-x)]}{\sin [\rho(b-a)]} f(b)
$$

for any $x \in[a, b]$.

If we add (1.2) with (2.2) we get

$$
\begin{aligned}
& f(x)+f(a+b-x) \\
& \leq \frac{\sin [\rho(b-x)]}{\sin [\rho(b-a)]} f(a)+\frac{\sin [\rho(x-a)]}{\sin [\rho(b-a)]} f(b) \\
& +\frac{\sin [\rho(x-a)]}{\sin [\rho(b-a)]} f(a)+\frac{\sin [\rho(b-x)]}{\sin [\rho(b-a)]} f(b) \\
& =\frac{\sin [\rho(b-x)]+\sin [\rho(x-a)]}{\sin [\rho(b-a)]} f(a) \\
& +\frac{\sin [\rho(b-x)]+\sin [\rho(x-a)]}{\sin [\rho(b-a)]} f(b) \\
& =\frac{\sin [\rho(b-x)]+\sin [\rho(x-a)]}{\sin [\rho(b-a)]}[f(a)+f(b)]
\end{aligned}
$$

for any $x \in[a, b]$.

Observe that

$$
\begin{aligned}
& \frac{\sin [\rho(b-x)]+\sin [\rho(x-a)]}{\sin [\rho(b-a)]} \\
& =\frac{2 \sin \left[\frac{\rho(b-a)}{2}\right] \cos \left[\rho\left(x-\frac{a+b}{2}\right)\right]}{2 \sin \left[\frac{\rho(b-a)}{2}\right] \cos \left[\frac{\rho(b-a)}{2}\right]}=\frac{\cos \left[\rho\left(x-\frac{a+b}{2}\right)\right]}{\cos \left[\frac{\rho(b-a)}{2}\right]}
\end{aligned}
$$

for any $x \in[a, b]$.

Using the equality (2.4) and dividing by 2 in (2.3) we get the second inequality in $(2.1)$. 
From (1.3) for $t=\frac{1}{2}$ and $a=u, b=v$ we get

$$
\begin{aligned}
f\left(\frac{u+v}{2}\right) & \leq \frac{\sin \left[\rho\left(\frac{v-u}{2}\right)\right]}{\sin [\rho(v-u)]} f(u)+\frac{\sin \left[\rho\left(\frac{v-u}{2}\right)\right]}{\sin [\rho(v-u)]} f(v) \\
& =\frac{\sin \left[\rho\left(\frac{v-u}{2}\right)\right]}{\sin [\rho(v-u)]}[f(u)+f(v)] \\
& =\frac{\sin \left[\rho\left(\frac{v-u}{2}\right)\right]}{2 \sin \left[\rho\left(\frac{v-u}{2}\right)\right] \cos \left[\rho\left(\frac{v-u}{2}\right)\right]}[f(u)+f(v)] \\
& =\frac{1}{\cos \left[\rho\left(\frac{v-u}{2}\right)\right]} \frac{f(u)+f(v)}{2}
\end{aligned}
$$

which implies that

$$
f\left(\frac{u+v}{2}\right) \cos \left[\rho\left(\frac{v-u}{2}\right)\right] \leq \frac{f(u)+f(v)}{2}
$$

for any $u, v \in I$.

Now, if we in (2.5) take $v=x$ and $u=a+b-x$, then we get the first inequality in $(2.1)$.

Remark 1. By taking $x=(1-t) a+t b$ in (2.1) we get the equivalent double inequality

$$
\begin{aligned}
f\left(\frac{a+b}{2}\right) \cos \left[\rho\left(t-\frac{1}{2}\right)(b-a)\right] & \\
\leq \frac{1}{2}[f((1-t) a+t b) & +f(t a+(1-t) b)] \\
& \leq \frac{f(a)+f(b)}{2} \frac{\cos \left[\rho\left(t-\frac{1}{2}\right)(b-a)\right]}{\cos \left[\frac{\rho(b-a)}{2}\right]}
\end{aligned}
$$

for any $a, b \in I$ with $0<|b-a|<\frac{\pi}{\rho}$ and $t \in[0,1]$.

We have the following Hermite-Hadamard type inequality that was obtained in a different and slightly more difficult manner in [2]

Theorem 1. Assume that the function $f: I \rightarrow \mathbb{R}$ is trigonometrically $\rho$-convex on I. Then for any $a, b \in I$ with $0<b-a<\frac{\pi}{\rho}$ we have

$$
\frac{2}{\rho} f\left(\frac{a+b}{2}\right) \sin \left[\frac{\rho(b-a)}{2}\right] \leq \int_{a}^{b} f(x) d x \leq \frac{f(a)+f(b)}{\rho} \tan \left[\frac{\rho(b-a)}{2}\right] .
$$

Proof. Integrating the inequality (2.1) over $x$ on $[a, b]$ we get

$$
\begin{aligned}
& f\left(\frac{a+b}{2}\right) \int_{a}^{b} \cos \left[\rho\left(x-\frac{a+b}{2}\right)\right] d x \\
& \leq \frac{1}{2}\left[\int_{a}^{b} f(x) d x+\int_{a}^{b} f(a+b-x) d x\right] \\
& \leq \frac{f(a)+f(b)}{2} \frac{\int_{a}^{b} \cos \left[\rho\left(x-\frac{a+b}{2}\right)\right] d x}{\cos \left[\frac{\rho(b-a)}{2}\right]}
\end{aligned}
$$


Since

$$
\begin{aligned}
\int_{a}^{b} \cos \left[\rho\left(x-\frac{a+b}{2}\right)\right] d x & =\left.\frac{1}{\rho} \sin \left[\rho\left(x-\frac{a+b}{2}\right)\right]\right|_{a} ^{b} \\
& =\frac{1}{\rho} \sin \left[\rho\left(b-\frac{a+b}{2}\right)\right]-\frac{1}{\rho} \sin \left[\rho\left(a-\frac{a+b}{2}\right)\right] \\
& =\frac{2}{\rho} \sin \left[\frac{\rho(b-a)}{2}\right]
\end{aligned}
$$

and by the change of variable $y=a+b-x$

$$
\int_{a}^{b} f(a+b-x) d x=\int_{a}^{b} f(y) d y
$$

hence by (2.8) we get (2.7).

Remark 2. The inequality (2.7) for $\rho=1$ was obtained in 2004 by M. Bessenyei in his Ph.D. Thesis [4, Corollary 2.13] in the context of Chebyshev system (cos, sin) .

We use the notation $\sec t=\frac{1}{\cos t}$ for $t \neq(2 k+1) \frac{\pi}{2}, k$ is an integer, to state the following weighted integral inequality of Hermite-Hadamard type:

Theorem 2. Assume that the function $f: I \rightarrow \mathbb{R}$ is trigonometrically $\rho$-convex on I. Then for any $a, b \in I$ with $0<b-a<\frac{\pi}{\rho}$ we have

$$
f\left(\frac{a+b}{2}\right) \leq \frac{1}{b-a} \int_{a}^{b} f(x) \sec \left[\rho\left(x-\frac{a+b}{2}\right)\right] d x \leq \frac{f(a)+f(b)}{2} .
$$

Proof. From (2.1) we have

$$
\begin{aligned}
f\left(\frac{a+b}{2}\right) \leq \frac{1}{2}[f(x)+f(a+b-x)] \sec & {\left[\rho\left(x-\frac{a+b}{2}\right)\right] } \\
\leq & \frac{f(a)+f(b)}{2} \sec \left[\frac{\rho(b-a)}{2}\right]
\end{aligned}
$$

for any $a, b \in I$ with $0<b-a<\frac{\pi}{\rho}$ and $x \in[a, b]$.

If we take the integral mean in $(2.10)$ we get

$$
\begin{array}{r}
f\left(\frac{a+b}{2}\right) \leq \frac{1}{2(b-a)} \int_{a}^{b}[f(x)+f(a+b-x)] \sec \left[\rho\left(x-\frac{a+b}{2}\right)\right] d x \\
\leq \frac{f(a)+f(b)}{2} \sec \left[\frac{\rho(b-a)}{2}\right]
\end{array}
$$

Using the change of variable $y=a+b-x$ we get

$$
\begin{aligned}
\int_{a}^{b} f(a+b-x) \sec \left[\rho\left(x-\frac{a+b}{2}\right)\right] d x & =\int_{a}^{b} f(y) \sec \left[\rho\left(\frac{a+b}{2}-y\right)\right] d y \\
& =\int_{a}^{b} f(y) \sec \left[\rho\left(y-\frac{a+b}{2}\right)\right] d y
\end{aligned}
$$

and by (2.11) we obtain the desired result (2.9).

The following weighted inequality also holds: 
Theorem 3. Assume that the function $f: I \rightarrow \mathbb{R}$ is trigonometrically $\rho$-convex on I. Then for any $a, b \in I$ with $0<b-a<\frac{\pi}{\rho}$ we have

$$
\begin{aligned}
& \frac{1}{2}\left[b-a+\frac{1}{\rho} \sin [\rho(b-a)]\right] f\left(\frac{a+b}{2}\right) \\
& \leq \int_{a}^{b} f(x) \cos \left[\rho\left(x-\frac{a+b}{2}\right)\right] d x \\
& \leq \frac{b-a+\frac{1}{\rho} \sin [\rho(b-a)]}{2 \cos \left[\frac{\rho(b-a)}{2}\right]} \frac{f(a)+f(b)}{2}
\end{aligned}
$$

Proof. If we multiply the inequality (2.1) by $\cos \left[\rho\left(x-\frac{a+b}{2}\right)\right] \geq 0$ we get

$$
\begin{aligned}
& f\left(\frac{a+b}{2}\right) \cos ^{2}\left[\rho\left(x-\frac{a+b}{2}\right)\right] \\
& \leq \frac{1}{2}[f(x)+f(a+b-x)] \cos \left[\rho\left(x-\frac{a+b}{2}\right)\right] \\
& \quad \leq \frac{f(a)+f(b)}{2} \frac{\cos ^{2}\left[\rho\left(x-\frac{a+b}{2}\right)\right]}{\cos \left[\frac{\rho(b-a)}{2}\right]}
\end{aligned}
$$

for any $x \in[a, b]$.

If we integrate this inequality over $x \in[a, b]$ we get

$$
\begin{aligned}
& f\left(\frac{a+b}{2}\right) \int_{a}^{b} \cos ^{2}\left[\rho\left(x-\frac{a+b}{2}\right)\right] d x \\
& \leq \frac{1}{2} \int_{a}^{b}[f(x)+f(a+b-x)] \cos \left[\rho\left(x-\frac{a+b}{2}\right)\right] d x \\
& \leq \frac{f(a)+f(b)}{2} \frac{\int_{a}^{b} \cos ^{2}\left[\rho\left(x-\frac{a+b}{2}\right)\right] d x}{\cos \left[\frac{\rho(b-a)}{2}\right]}
\end{aligned}
$$

Since

$$
\int_{a}^{b} \cos ^{2}\left[\rho\left(x-\frac{a+b}{2}\right)\right] d x=\frac{1}{2}\left[(b-a)+\int_{a}^{b} \cos \left[2 \rho\left(x-\frac{a+b}{2}\right)\right] d x\right]
$$

and

$$
\begin{aligned}
\int_{a}^{b} \cos \left[2 \rho\left(x-\frac{a+b}{2}\right)\right] d x & =\left.\frac{1}{2 \rho} \sin \left[2 \rho\left(x-\frac{a+b}{2}\right)\right]\right|_{a} ^{b} \\
& =\frac{1}{2 \rho} \sin \left[2 \rho\left(b-\frac{a+b}{2}\right)\right]-\frac{1}{2 \rho} \sin \left[2 \rho\left(a-\frac{a+b}{2}\right)\right] \\
& =\frac{1}{\rho} \sin [\rho(b-a)]
\end{aligned}
$$

hence

$$
\int_{a}^{b} \cos ^{2}\left[\rho\left(x-\frac{a+b}{2}\right)\right] d x=\frac{1}{2}\left[(b-a)+\frac{1}{\rho} \sin [\rho(b-a)]\right] .
$$


Also, by the change of variable $y=a+b-x$ we have

$$
\int_{a}^{b} f(a+b-x) \cos \left[\rho\left(x-\frac{a+b}{2}\right)\right] d x=\int_{a}^{b} f(y) \cos \left[\rho\left(y-\frac{a+b}{2}\right)\right] d x
$$

and by (2.13) we get (2.12).

\section{Related Results}

Theorem 4. Assume that the function $f: I \rightarrow \mathbb{R}$ is trigonometrically $\rho$-convex on I. Then for any $a, b \in I$ with $0<b-a<\frac{\pi}{\rho}$ we have

$$
\begin{aligned}
\int_{a}^{b} f(t) d t \geq & \frac{2}{\rho} \sin \left[\rho\left(\frac{b-a}{2}\right)\right] \\
& \times\left\{f(x) \cos \left[\rho\left(x-\frac{a+b}{2}\right)\right]+K_{x, f} \sin \left[\rho\left(\frac{a+b}{2}-x\right)\right]\right\}
\end{aligned}
$$

for any $x \in[a, b]$, where $K_{x, f} \in\left[f_{-}^{\prime}(x), f_{+}^{\prime}(x)\right]$.

If $f$ is differentiable in $x$, then

$$
\begin{aligned}
\int_{a}^{b} f(t) d t \geq & \frac{2}{\rho} \sin \left[\rho\left(\frac{b-a}{2}\right)\right] \\
& \times\left\{f(x) \cos \left[\rho\left(x-\frac{a+b}{2}\right)\right]+f^{\prime}(x) \sin \left[\rho\left(\frac{a+b}{2}-x\right)\right]\right\}
\end{aligned}
$$

Proof. If we take the integral over $y \in[a, b]$ in the gradient inequality (1.4) we get

$$
\int_{a}^{b} f(y) d y \geq f(x) \int_{a}^{b} \cos [\rho(y-x)] d y+K_{x, f} \int_{a}^{b} \sin [\rho(y-x)] d y
$$

where $K_{x, f} \in\left[f_{-}^{\prime}(x), f_{+}^{\prime}(x)\right]$.

Observe that

$$
\begin{aligned}
\int_{a}^{b} \cos [\rho(y-x)] d y & =\left.\frac{1}{\rho} \sin [\rho(y-x)]\right|_{a} ^{b}=\frac{1}{\rho} \sin [\rho(b-x)]+\frac{1}{\rho} \sin [\rho(x-a)] \\
& =\frac{2}{\rho} \sin \left[\rho\left(\frac{b-a}{2}\right)\right] \cos \left[\rho\left(x-\frac{a+b}{2}\right)\right]
\end{aligned}
$$

and

$$
\begin{aligned}
\int_{a}^{b} \sin [\rho(y-x)] d y & =-\left.\frac{1}{\rho} \cos [\rho(y-x)]\right|_{a} ^{b}=-\frac{1}{\rho} \cos [\rho(b-x)]+\frac{1}{\rho} \cos [\rho(a-x)] \\
& =\frac{1}{\rho}[\cos [\rho(a-x)]-\cos [\rho(b-x)]] \\
& =-\frac{2}{\rho} \sin \left[\rho\left(\frac{a+b}{2}-x\right)\right] \sin \left[\rho\left(\frac{a-b}{2}\right)\right] \\
& =\frac{2}{\rho} \sin \left[\rho\left(\frac{b-a}{2}\right)\right] \sin \left[\rho\left(\frac{a+b}{2}-x\right)\right]
\end{aligned}
$$

or any $x \in[a, b]$.

By utilising the inequality (3.3) we then get (3.1).

Remark 3. We observe that, if we take $x=\frac{a+b}{2}$ in (3.1), then we recapture the first inequality in (2.7). 
Theorem 5. Assume that the function $f: I \rightarrow \mathbb{R}$ is trigonometrically $\rho$-convex on I. Then for any $a, b \in I$ with $0<b-a<\frac{\pi}{\rho}$ we have

$$
\int_{a}^{b} f(t) \sec [\rho(t-x)] d t \geq f(x)(b-a)+\frac{1}{\rho} K_{x, f} \ln \left(\frac{\cos [\rho(x-a)]}{\cos [\rho(b-x)]}\right)
$$

for any $x \in[a, b]$, where $K_{x, f} \in\left[f_{-}^{\prime}(x), f_{+}^{\prime}(x)\right]$.

If $f$ is differentiable in $x$, then

$$
\int_{a}^{b} f(t) \sec [\rho(t-x)] d t \geq f(x)(b-a)+\frac{1}{\rho} f^{\prime}(x) \ln \left(\frac{\cos [\rho(x-a)]}{\cos [\rho(b-x)]}\right)
$$

for any $x \in[a, b]$.

Proof. We have by the gradient inequality (1.4) that

$$
f(y) \sec [\rho(y-x)] \geq f(x)+K_{x, f} \tan [\rho(y-x)]
$$

for any $x, y \in[a, b]$.

If we integrate (3.6) over $y \in[a, b]$, then we get

$$
\int_{a}^{b} f(y) \sec [\rho(y-x)] d y \geq f(x)(b-a)+K_{x, f} \int_{a}^{b} \tan [\rho(y-x)] d y
$$

for any $x \in[a, b]$.

Since

$$
\begin{aligned}
\int_{a}^{b} \tan [\rho(y-x)] d y & =\int_{a}^{b} \frac{\sin [\rho(y-x)]}{\cos [\rho(y-x)]} d y \\
& =-\frac{1}{\rho} \int_{a}^{b} \frac{1}{\cos [\rho(y-x)]} d(\cos [\rho(y-x)]) \\
& =-\left.\frac{1}{\rho} \ln (\cos [\rho(y-x)])\right|_{a} ^{b} \\
& =-\frac{1}{\rho} \ln (\cos [\rho(b-x)])+\frac{1}{\rho} \ln (\cos [\rho(a-x)]) \\
& =\frac{1}{\rho} \ln \left(\frac{\cos [\rho(x-a)]}{\cos [\rho(b-x)]}\right)
\end{aligned}
$$

for any $x \in[a, b]$, hence by (3.7) we get (3.4).

Remark 4. We observe that, if we take $x=\frac{a+b}{2}$ in (3.4), then we recapture the first inequality in (2.9).

From a different perspective, we have:

Theorem 6. Assume that the function $f: I \rightarrow \mathbb{R}$ is trigonometrically $\rho$-convex on I. Then for any $a, b \in I$ with $0<b-a<\frac{\pi}{\rho}$ we have

$$
\begin{aligned}
\frac{1}{\rho+1}\{f(y)(b-a)+f(b) \sin [\rho(b-y)]+f(a) & \sin [\rho(y-a)]\} \\
& \geq \int_{a}^{b} f(x) \cos [\rho(x-y)] d x
\end{aligned}
$$

for any $y \in[a, b]$. 
In particular, for $y=\frac{a+b}{2}$, we get

$$
\begin{aligned}
& \frac{1}{\rho+1}\left\{f\left(\frac{a+b}{2}\right)(b-a)+[f(b)+f(a)] \sin \left[\rho\left(\frac{b-a}{2}\right)\right]\right\} \\
& \geq \int_{a}^{b} f(x) \cos \left[\rho\left(x-\frac{a+b}{2}\right)\right] d x
\end{aligned}
$$

Proof. The function $f: I \rightarrow \mathbb{R}$ is differentiable almost everywhere and we have

$$
f(y) \geq f(x) \cos [\rho(y-x)]+f^{\prime}(x) \sin [\rho(y-x)]
$$

for almost every $x \in[a, b]$ where $y \in[a, b]$.

If we integrate the inequality over $x \in[a, b]$ we get

$$
f(y)(b-a) \geq \int_{a}^{b} f(x) \cos [\rho(y-x)] d x+\int_{a}^{b} f^{\prime}(x) \sin [\rho(y-x)] d x
$$

for any $y \in[a, b]$.

Using the integration by parts, we have

$$
\begin{aligned}
\int_{a}^{b} f^{\prime}(x) \sin [\rho(y-x)] d x & =\left.f(x) \sin [\rho(y-x)]\right|_{a} ^{b}+\rho \int_{a}^{b} f(x) \cos [\rho(y-x)] d x \\
& =-f(b) \sin [\rho(b-y)]-f(a) \sin [\rho(y-a)] \\
& +\rho \int_{a}^{b} f(x) \cos [\rho(y-x)] d x
\end{aligned}
$$

and by (3.11) we get

$$
\begin{aligned}
f(y)(b-a) & \geq-f(b) \sin [\rho(b-y)]-f(a) \sin [\rho(y-a)] \\
& +(\rho+1) \int_{a}^{b} f(x) \cos [\rho(y-x)] d x,
\end{aligned}
$$

for any $y \in[a, b]$, which is equivalent to the desired result (3.8).

\section{Some Inequalities for Special Means}

Recall the following special means:

(1) The arithmetic mean

$$
A=A(a, b):=\frac{a+b}{2}, \quad a, b \geq 0 ;
$$

(2) The geometric mean:

$$
G=G(a, b):=\sqrt{a b}, \quad a, b \geq 0 ;
$$

(3) The harmonic mean:

$$
H=H(a, b):=\frac{2}{\frac{1}{a}+\frac{1}{b}}, \quad a, b \geq 0
$$

(4) The logarithmic mean:

$$
L=L(a, b):=\left\{\begin{array}{ll}
a & \text { if } a=b \\
\frac{b-a}{\ln b-\ln a} & \text { if } a \neq b
\end{array} a, b>0 ;\right.
$$


(5) The identric mean:

$$
I:=I(a, b)=\left\{\begin{array}{ll}
a & \text { if } a=b \\
\frac{1}{e}\left(\frac{b^{b}}{a^{a}}\right)^{\frac{1}{b-a}} & \text { if } a \neq b
\end{array} \quad a, b>0 ;\right.
$$

(6) The $p$-logarithmic mean:

$$
L_{p}=L_{p}(a, b):= \begin{cases}{\left[\frac{b^{p+1}-a^{p+1}}{(p+1)(b-a)}\right]^{\frac{1}{p}}} & \text { if } a \neq b \\ a & \text { if } a=b\end{cases}
$$

where $p \in \mathbb{R} \backslash\{-1,0\}$ and $a, b>0$.

It is well known that $L_{p}$ is monotonic nondecreasing over $p \in \mathbb{R}$ with $L_{-1}:=L$ and $L_{0}:=I$.

In particular, we have the inequalities

$$
H \leq G \leq L \leq I \leq A
$$

Consider the function $f:(0, \infty) \rightarrow(0, \infty), f(x)=x^{p}$ with $p \in \mathbb{R} \backslash\{0\}$. If $p \in(-\infty, 0) \cup[1, \infty)$ the function is convex and therefore trigonometrically $\rho$-convex for any $\rho>0$. If $p \in(0,1)$ then the function is concave and

$$
f^{\prime \prime}(x)+\rho^{2} f(x)=\rho^{2} x^{p}-p(1-p) x^{p-2}=\rho^{2} x^{p-2}\left(x^{2}-\frac{p(1-p)}{\rho^{2}}\right), x>0 .
$$

This shows that for $p \in(0,1)$ and $\rho>0$ the function $f(x)=x^{p}$ is trigonometrically $\rho$-convex on $\left(\frac{1}{\rho} \sqrt{p(1-p)}, \infty\right)$ and trigonometrically $\rho$-concave on $\left(0, \frac{1}{\rho} \sqrt{p(1-p)}\right)$.

For $p \in \mathbb{R} \backslash\{-1,0\}$ and $0<a<b$ we have

$$
\frac{1}{b-a} \int_{a}^{b} x^{p} d x=L_{p}^{p}(a, b) .
$$

Now, by applying the inequality (2.7) we have for $\rho>0$ and $0<b-a<\frac{\pi}{\rho}$ that

$$
\frac{2}{\rho} A^{p}(a, b) \sin \left[\frac{\rho(b-a)}{2}\right] \leq(b-a) L_{p}^{p}(a, b) \leq \frac{2}{\rho} A\left(a^{p}, b^{p}\right) \tan \left[\frac{\rho(b-a)}{2}\right]
$$

where $p \in(-\infty, 0) \cup[1, \infty)$ and $0<a<b<\infty$ or $p \in(0,1)$ and $\frac{1}{\rho} \sqrt{p(1-p)}<$ $a<b<\infty$. If $p \in(0,1)$ and $0<a<b<\frac{1}{\rho} \sqrt{p(1-p)}$ then the sign of inequality reverses in (4.2).

If we use the inequality (2.9), then we have for $\rho>0$ and $0<b-a<\frac{\pi}{\rho}$ that

$$
A^{p}(a, b) \leq \frac{1}{b-a} \int_{a}^{b} x^{p} \sec \left[\rho\left(x-\frac{a+b}{2}\right)\right] d x \leq A\left(a^{p}, b^{p}\right)
$$

where $p \in(-\infty, 0) \cup[1, \infty)$ and $0<a<b<\infty$ or $p \in(0,1)$ and $\frac{1}{\rho} \sqrt{p(1-p)}<$ $a<b<\infty$. If $p \in(0,1)$ and $0<a<b<\frac{1}{\rho} \sqrt{p(1-p)}$ then the sign of inequality reverses in $(4.2)$. 
Finally, if we use the inequality (2.12), then we have for $\rho>0$ and $0<b-a<\frac{\pi}{\rho}$ that

$$
\begin{aligned}
& \frac{1}{2}\left[b-a+\frac{1}{\rho} \sin [\rho(b-a)]\right] A^{p}(a, b) \\
& \leq \int_{a}^{b} x^{p} \cos \left[\rho\left(x-\frac{a+b}{2}\right)\right] d x \\
& \quad \leq \frac{b-a+\frac{1}{\rho} \sin [\rho(b-a)]}{2 \cos \left[\frac{\rho(b-a)}{2}\right]} A\left(a^{p}, b^{p}\right),
\end{aligned}
$$

where $p \in(-\infty, 0) \cup[1, \infty)$ and $0<a<b<\infty$ or $p \in(0,1)$ and $\frac{1}{\rho} \sqrt{p(1-p)}<$ $a<b<\infty$. If $p \in(0,1)$ and $0<a<b<\frac{1}{\rho} \sqrt{p(1-p)}$ then the sign of inequality reverses in (4.2).

Consider the concave function $f:(0, \infty) \rightarrow \mathbb{R}, f(x)=\ln x$. We observe that

$$
g(x):=f^{\prime \prime}(x)+\rho^{2} f(x)=\rho^{2} \ln x-\frac{1}{x^{2}}, x>0 .
$$

We have

$$
g^{\prime}(x)=\frac{2+\rho^{2} x^{2}}{x^{2}}>0 \text { for } x>0
$$

and

$$
\lim _{x \rightarrow 0+} g(x)=-\infty, \quad \lim _{x \rightarrow \infty} g(x)=\infty
$$

showing that the function $g$ is strictly increasing on $(0, \infty)$ and the equation $g(x)=$ 0 has a unique solution. Therefore $g(x)<0$ for $x \in\left(0, x_{\rho}\right)$ and $g(x)>0$ for $x \in\left(x_{\rho}, \infty\right)$, where $x_{\rho}$ is the unique solution of the equation $\ln x=\frac{1}{\rho^{2} x^{2}}$.

In conclusion, if $\rho>0$, then the function $f(x)=\ln x$ is trigonometrically $\rho$ concave on $\left(0, x_{\rho}\right)$ and trigonometrically $\rho$-convex on $\left(x_{\rho}, \infty\right)$.

Since

$$
\frac{1}{b-a} \int_{a}^{b} \ln x d x=\frac{b \ln b-a \ln a-(b-a)}{b-a}=\ln I(a, b),
$$

where $I(a, b)$ is the identric mean, then by $(2.7)$ we get for $\rho>0$ and $0<b-a<\frac{\pi}{\rho}$ that

$$
[A(a, b)]^{\frac{2}{\rho} \sin \left[\frac{\rho(b-a)}{2}\right]} \leq[I(a, b)]^{b-a} \leq[G(a, b)]^{\frac{2}{\rho} \tan \left[\frac{\rho(b-a)}{2}\right]}
$$

provided $[a, b] \subset\left(x_{\rho}, \infty\right)$ where $x_{\rho}$ is the unique solution of the equation $\ln x=\frac{1}{\rho^{2} x^{2}}$.

If $[a, b] \subset\left(0, x_{\rho}\right)$, the inequality in $(4.5)$ is reversed.

Other similar Hermite-Hadamard type inequalities may be stated, however the details are left to the interested reader.

\section{REFERENCES}

[1] M. S. S. Ali, On certain properties of trigonometrically $\rho$-convex functions, Advances in Pure Mathematics, 2012, 2, 337-340 http://dx.doi.org/10.4236/apm.2012.25047.

[2] M. S. S. Ali, On Hadamard's inequality for trigonometrically $\rho$-convex functions," accepted to appear in Theoretical Mathematics \& Applications., March, 2013.

[3] E. F. Beckenbach, Convex functions, Bulletin of the American Mathematical Society, Vol. 54, No. 5, 1948, pp. 439-460. doi:10.1090/S0002-9904-1948-08994-7

[4] M. Bessenyei, Hermite-Hadamard-type inequalities for generalized convex functions, PhD Thesis, Univ. Debrecen, Hungary, 2004. RGMIA Monograps, [http://rgmia.org/papers/monographs/dissertatio.pdf] 
[5] F. F. Bonsall, The characterization of generalized convex functions, The Quarterly Journal of Mathematics Oxford Series, Vol. 1, 1950, pp. 100-111. doi:10.1093/qmath/1.1.100

[6] A. M. Bruckner and E. Ostrow, Some functions classes related to the class of convex functions," Pacific Journal of Mathematics, Vol. 12, 1962, pp. 1203-1215.

[7] S. S. Dragomir, Ostrowski type inequalities for Lebesgue integral: a survey of recent results, Australian J. Math. Anal. Appl., Volume 14, Issue 1, Article 1, pp. 1-287, 2017. [Online http: //ajmaa.org/cgi-bin/paper.pl?string=v14n1/V14I1P1.tex] .

[8] S. S. Dragomir and C. E. M. Pearce, Selected Topics on HermiteHadamard Inequalities and Applications, RGMIA Monographs, 2000. [Online http://rgmia.org/monographs/hermite_hadamard.html] .

[9] J. W. Green, Support, Convergence, and Differentiability Properties of Generalized Convex Functions, Proceedings of the American Mathematical Society, Vol. 4, No. 3, 1953, pp. 391396. doi:10.1090/S0002-9939-1953-0056039-2

[10] B. Ya. Levin, Lectures on Entire Functions, American Mathematical Society, 1996.

[11] L. S. Maergoiz, Asymptotic Characteristics of Entire Functions and Their Applications in Mathematics and Biophysics, Kluwer Academic Publishers, New York, 2003.

[12] M. J. Miles, An extremum property of convex functions, American Mathematical Monthly, Vol. 76, 1969, pp. 921-922. doi:10.2307/2317948

[13] M. M. Peixoto, On the existence of derivatives of generalized convex functions, Summa Brasilian Mathematics, Vol. 2, No. 3, 1948, pp. 35-42.

[14] M. M. Peixoto, Generalized convex functions and second order differential inequlities, Bulletin of the American Mathematical Society, Vol. 55, No. 6, 1949, pp. 563- 572. doi:10.1090/S00029904-1949-09246-7

[15] A. W. Roberts and D. E. Varberg, Convex Functions, Academic Press, New York, 1973.

${ }^{1}$ Mathematics, College of Engineering \& Science, Victoria University, PO Box 14428, Melbourne City, MC 8001, Australia.

Email address: sever.dragomir@vu.edu.au

$U R L:$ http://rgmia.org/dragomir

${ }^{2}$ DSt-NRF Centre of Excellence, in the Mathematical and Statistical Sciences, School of Computer Science \& Applied Mathematics, University of the Witwatersrand, Private Bag 3, Johannesburg 2050, South Africa 